\title{
An Approximate Rolle's Theorem for Polynomials of Degree Four in a Hilbert Space
}

By

\author{
Jesús FERRER*
}

\begin{abstract}
We show that the fourth degree polynomials that satisfy Rolle's Theorem in the unit ball of a real Hilbert space are dense in the space of polynomials that vanish in the unit sphere. As a consequence, we obtain a sort of approximate Rolle's Theorem for those polynomials.
\end{abstract}

\section{$\S 1$. Introduction}

S. Shkarin showed in [5] that Rolle's Theorem fails in general for continuous polynomials, even of degree four, in the unit ball of a Hilbert space. In [4], we generalized Shkarin's example finding a class of four degree polynomials for which the result does not hold. In the same paper, we gave some sufficient conditions for this type of polynomials to satisfy the theorem.

The purpose of this paper is to show that the polynomials (always assumed to be continuous and of degree four) that satisfy Rolle's Theorem form a dense subset of those vanishing on the unit sphere.

More specifically, recalling that a continuous polynomial of the fourth degree $P$ which vanishes on the unit sphere of a real Hilbert space $X$ has the form

$$
P(x)=\left(1-\|x\|^{2}\right) Q(x), \quad Q(x)=\langle A x, x\rangle+2\langle\varphi, x\rangle+k,
$$

with $A$ being a non-zero bounded self-adjoint operator in $X, \varphi \in X, k \in \mathbb{R}$, we show that, for every $\varepsilon>0$, there is a polynomial $P_{\varepsilon}$ which satisfies Rolle's

\footnotetext{
Communicated by H. Okamoto. Received April 14, 2003. Revised September 16, 2003. 2000 Mathematics Subject Classification(s): 49J50, 49J52.

Key words: Rolle's Theorem, Hilbert spaces, polynomials

The author has been partially supported by MCyT and FEDER Project BFM2002-01423.

*Departament d'Anàlisi Matemàtica, Universitat de València, 46100 Burjassot, Spain. e-mail: Jesus.Ferrer@uv.es
} 
Theorem (i.e., there is a vector $x_{0} \in X$ with $\left\|x_{0}\right\|<1$ and the Fréchet-derivative is such that $\left.P_{\varepsilon}^{\prime}\left(x_{0}\right)=0\right)$ and at the same time $\left\|P-P_{\varepsilon}\right\| \leq \varepsilon$. As a by-product of this density result, we also show that there exists an element $x_{\varepsilon} \in X$ such that $\left\|x_{\varepsilon}\right\|<1$ and $\left\|P^{\prime}\left(x_{\varepsilon}\right)\right\| \leq \varepsilon$. We must say that another version of an approximate Rolle's Theorem is given in [2], although with a different approach.

We must also recall that, if $A$ is a compact self-adjoint operator in $X$, then, since the polynomial $Q(x)$ is weakly continuous, see [1], and the factor $1-\|x\|^{2}$ is weakly upper semicontinuous, it follows, as showed in [3], that the polynomial $P(x)$ satisfies Rolle's Theorem.

For the sake of commodity, the symbols $\mathcal{P}_{4}(X)$ and $\mathcal{R}_{4}(X)$ will denote the Banach space of at most four degree continuous polynomials with zero-value in the unit sphere, endowed with its usual norm, that is,

$$
\|P\|=\sup \{|P(x)|:\|x\| \leq 1\},
$$

and the subset of polynomials satisfying Rolle's Theorem, respectively.

\section{§2. Density of the Polynomials that Satisfy Rolle's Theorem}

Let $P$ be an element of the space $\mathcal{P}_{4}(X)$. Then, as we mentioned before, $P(x)=\left(1-\|x\|^{2}\right) Q(x)$, where $Q(x)=\langle A x, x\rangle+2\langle\varphi, x\rangle+k$. We assume that $A \neq 0$, otherwise it may be easily seen that $P \in \mathcal{R}_{4}(X)$. Let us suppose that, for real numbers $\lambda$ in a certain domain, we may find a vector $x(\lambda)$ in $X$ such that the vector equation $(I-\lambda A) x(\lambda)=\lambda \varphi$ holds. For such $\lambda$, let us consider the real-valued function

$$
h(\lambda):=\|x(\lambda)\|^{2}+\lambda Q(x(\lambda)) .
$$

Then, calculating the derivatives $P^{\prime}(x)$ and $Q^{\prime}(x)$, it is clear that a sufficient condition to guarantee that $P \in \mathcal{R}_{4}(X)$ is to show that there is a certain $\lambda_{0}$ for which

$$
\left\|x\left(\lambda_{0}\right)\right\|<1, \quad h\left(\lambda_{0}\right)=1 .
$$

Thus, our problem may be reduced to the one to find an appropriate domain for $\lambda \in \mathbb{R}$, then seeking the conditions before stated. With this in mind, we define the following values (possibly infinite):

$$
\begin{aligned}
& r_{A}:=\sup \{\lambda>0: I-\mu A \text { is a topological isomorphism, } 0<\mu<\lambda\}, \\
& s_{A}:=\inf \{\lambda<0: I-\mu A \text { is a topological isomorphism, } \lambda<\mu<0\}, \\
& t_{A}:=\min \left\{r_{A},-s_{A}\right\} .
\end{aligned}
$$

Now, since $A$ is non-zero, bounded and self-adjoint, we know that $t_{A}$ is always finite and that either $I-t_{A} A$, or $I+t_{A} A$, cannot be an isomorphism. 
It is also clear that

$$
] \frac{-1}{\|A\|}, \frac{1}{\|A\|}[\subset]-t_{A}, t_{A}[\subset] s_{A}, r_{A}[.
$$

Hence, by applying the implicit function theorem to the equation

$$
(I-\lambda A) x=\lambda \varphi
$$

one obtains an infinitely differentiable function $x(\lambda), \lambda \in] s_{A}, r_{A}[$, satisfying this equality. Moreover, the real-valued functions $\langle\varphi, x(\lambda)\rangle$ and $h(\lambda)$ are both infinitely differentiable in that interval. We proceed next to show some auxiliary details that will be used later. We shall be assuming always that $\varphi \neq 0$, otherwise $P^{\prime}(0)=0$.

Lemma 1. The function $\langle\varphi, x(\lambda)\rangle$ increases strictly in $] s_{A}, r_{A}[$.

Proof. For $\lambda \in] s_{A}, r_{A}\left[, \lambda \neq 0\right.$, taking derivatives in the equality $\left(\frac{1}{\lambda} I-\right.$ A) $x(\lambda)=\varphi$, we obtain

$$
\left(\frac{1}{\lambda} I-A\right) x^{\prime}(\lambda)=\frac{1}{\lambda^{2}} x(\lambda) .
$$

But, since $\frac{1}{\lambda} I-A$ is self-adjoint, we have

$$
\begin{aligned}
\frac{d}{d \lambda}\langle\varphi, x(\lambda)\rangle & =\left\langle\varphi, x^{\prime}(\lambda)\right\rangle=\left\langle\left(\frac{1}{\lambda} I-A\right) x(\lambda), x^{\prime}(\lambda)\right\rangle \\
& =\left\langle x(\lambda),\left(\frac{1}{\lambda} I-A\right) x^{\prime}(\lambda)\right\rangle=\frac{\|x(\lambda)\|^{2}}{\lambda^{2}}>0
\end{aligned}
$$

after noticing that $x(\lambda)=0$ would imply $\lambda=0$.

Lemma 2. If $k \notin\left[\frac{1}{s_{A}}, \frac{1}{r_{A}}\right]$, then $P \in \mathcal{R}_{4}(X)$.

Proof. We assume $k>\frac{1}{r_{A}}$, the other alternative can be shown in an analogous way. For $\lambda \in] 0, r_{A}[$, making use of the mean value theorem, we get hold of a certain $\mu \in] 0, \lambda[$ such that

$$
\langle\varphi, x(\lambda)\rangle=\frac{\|x(\mu)\|^{2}}{\mu^{2}} \cdot \lambda .
$$

Thus, doing straightforward computations,

$$
\begin{aligned}
h(\lambda) & =\|x(\lambda)\|^{2}+\lambda Q(x(\lambda))=\|x(\lambda)\|^{2}+\lambda[\langle A x(\lambda), x(\lambda)\rangle+2\langle\varphi, x(\lambda)\rangle+k] \\
& =\|x(\lambda)\|^{2}+\lambda[\langle A x(\lambda)+\varphi, x(\lambda)\rangle+\langle\varphi, x(\lambda)\rangle+k] \\
& =2\|x(\lambda)\|^{2}+\lambda(\langle\varphi, x(\lambda)\rangle+k) \\
& =2\|x(\lambda)\|^{2}+\frac{\lambda^{2}}{\mu^{2}}\|x(\mu)\|^{2}+k \lambda>k \lambda .
\end{aligned}
$$


In particular, for $\lambda=1 / k \in] 0, r_{A}$ [, we have that $h(1 / k)>1$, and so, since $h(0)=0$, continuity assures the existence of $\left.\lambda_{0} \in\right] 0,1 / k\left[\right.$ such that $h\left(\lambda_{0}\right)=1$. Clearly,

$$
\left\|x\left(\lambda_{0}\right)\right\|^{2}<\frac{1}{2} h\left(\lambda_{0}\right)=\frac{1}{2}<1,
$$

and, after the considerations above mentioned, it follows that $P \in \mathcal{R}_{4}(X)$.

Lemma 3. If either $\frac{1}{t_{A}}$, or $-\frac{1}{t_{A}}$, is an eigenvalue of $A$, then $P \in$ $\mathcal{R}_{4}(X)$.

Proof. We assume that $\frac{1}{t_{A}}$ is an eigenvalue, the other case may be shown in an analogous manner. Let $z$ be a non-zero element of $\operatorname{Ker}\left(I-t_{A} A\right)$. We also assume that $k \in\left[\frac{1}{s_{A}}, \frac{1}{r_{A}}\right]$, otherwise Lemma 2 applies. We now take into consideration the following alternatives:

One: There is $\left.\lambda_{0} \in\right] 0, t_{A}$ [ such that $h\left(\lambda_{0}\right)=1$. Then, since, after Lemma $1, \lambda_{0}\left\langle\varphi, x\left(\lambda_{0}\right)\right\rangle$ is positive, we have

$$
\begin{aligned}
\left\|x\left(\lambda_{0}\right)\right\|^{2} & =\frac{1}{2}\left(1-\lambda_{0}\left\langle\varphi, x\left(\lambda_{0}\right)\right\rangle-k \lambda_{0}\right) \\
& <\frac{1}{2}\left(1-k \lambda_{0}\right) \leq \frac{1}{2}\left(1+\left|k \lambda_{0}\right|\right) \\
& \leq \frac{1}{2}\left(1+|k| \cdot t_{A}\right) \leq 1 .
\end{aligned}
$$

So, $P \in \mathcal{R}_{4}(X)$.

Two. For all $\lambda \in] 0, t_{A}[, \quad h(\lambda)<1$. Then, we have that the set $\{x(\lambda)$ : $\left.0<\lambda<t_{A}\right\}$ is bounded in $X$, and so we may find a sequence $\left(\lambda_{j}\right)_{j=1}^{\infty}$ in $] 0, t_{A}$ [ and a vector $\phi$ in $X$ such that $\lim _{j} \lambda_{j}=t_{A}$, and the vector sequence $\left(x\left(\lambda_{j}\right)\right)_{j=1}^{\infty}$ converges weakly to $\phi$. Hence, taking weak limits in the equality

$$
\left(I-\lambda_{j} A\right) x\left(\lambda_{j}\right)=\lambda_{j} \varphi, \quad j \geq 1,
$$

we obtain

$$
\left(I-t_{A} A\right) \phi=t_{A} \varphi
$$

and also, remembering that $h(\lambda)=2\|x(\lambda)\|^{2}+\lambda(\langle\varphi, x(\lambda)\rangle+k)$, it follows that

$$
1 \geq \sup _{j} h\left(\lambda_{j}\right) \geq 2\|\phi\|^{2}+t_{A}(\langle\varphi, \phi\rangle+k) .
$$

For $t \in \mathbb{R}$, the vector $\phi+t z$ is such that

$$
\left(I-t_{A} A\right)(\phi+t z)=\left(I-t_{A} A\right) \phi=t_{A} \varphi,
$$

that is, $\phi+t z$ is a solution of the vector equation $(I-\lambda A) x=\lambda \varphi$, for $\lambda=t_{A}$. 
Considering now the real-valued function

$$
h_{1}(t):=\|\phi+t z\|^{2}+t_{A} \cdot Q(\phi+t z), t \in \mathbb{R} .
$$

(Notice that it may be regarded as a continuation of $h(\lambda)=\|x(\lambda)\|^{2}+\lambda Q(x(\lambda)$ ) for $\lambda=t_{A}$ ), we show that $h_{1}(t)$ behaves similarly to $h(\lambda)$.

Recalling that

$$
\langle\varphi, z\rangle=\frac{1}{t_{A}}\left\langle\left(I-t_{A} A\right) \phi, z\right\rangle=\frac{1}{t_{A}}\left\langle\phi,\left(I-t_{A} A\right) z\right\rangle=0,
$$

and $A(\phi+t z)=\frac{1}{t_{A}}(\phi+t z)-\varphi$, we have that

$$
\begin{aligned}
h_{1}(t) & =\|\phi+t z\|^{2}+t_{A}(\langle A(\phi+t z), \phi+t z\rangle+2\langle\varphi, \phi+t z\rangle+k) \\
& =\|\phi+t z\|^{2}+t_{A}\left(\frac{1}{t_{A}}\|\phi+t z\|^{2}+\langle\varphi, \phi+t z\rangle+k\right) \\
& =2\|\phi+t z\|^{2}+t_{A}(\langle\varphi, \phi\rangle+k) .
\end{aligned}
$$

Therefore, since, after $(1), h_{1}(0)=2\|\phi\|^{2}+t_{A}(\langle\varphi, \phi\rangle+k) \leq 1, \lim _{t \rightarrow \infty} h_{1}(t)=$ $+\infty$, and $h_{1}(t)$ is continuous, we can find a non-negative value $t_{0}$ such that $h_{1}\left(t_{0}\right)=1$. But, since $\langle\varphi, x(\lambda)\rangle$ is increasing, we know that $\langle\varphi, \phi\rangle=\lim _{j}$ $\left\langle\varphi, x\left(\lambda_{j}\right)\right\rangle>0$. Hence, setting $x_{0}:=\phi+t_{0} z$, it follows that

$$
\left\|x_{0}\right\|^{2}=\left\|\phi+t_{0} z\right\|^{2}=\frac{1}{2}\left(1-t_{A}\langle\varphi, \phi\rangle-t_{A} k\right)<\frac{1}{2}\left(1+t_{A}|k|\right) \leq 1,
$$

and

$$
\begin{aligned}
P^{\prime}\left(x_{0}\right) & =2\left[\left(1-\left\|x_{0}\right\|^{2}\right)\left(A x_{0}+\varphi\right)-Q\left(x_{0}\right) x_{0}\right] \\
& =2\left[\frac{1}{t_{A}}\left(1-\left\|x_{0}\right\|^{2}\right) x_{0}-Q\left(x_{0}\right) x_{0}\right] \\
& =\frac{2}{t_{A}}\left(1-\left\|x_{0}\right\|^{2}-t_{A} Q\left(x_{0}\right)\right) x_{0}=\frac{2}{t_{A}}\left(1-h_{1}\left(t_{0}\right)\right) x_{0}=0 .
\end{aligned}
$$

Lemma 4. If $\varphi \notin \operatorname{Range}\left(I-t_{A} A\right) \cap \operatorname{Range}\left(I+t_{A} A\right)$, then $P \in \mathcal{R}_{4}(X)$.

Proof. Seeking a contradiction, let us assume that $P \notin \mathcal{R}_{4}(X)$. Then, after Lemma $2, k \in\left[\frac{1}{s_{A}}, \frac{1}{r_{A}}\right]$, and so, for $\left.\lambda \in\right]-t_{A}, t_{A}[, h(\lambda)<1$. Consequently, proceeding as in the previous lemma, since the set $\left\{x(\lambda):|\lambda|<t_{A}\right\}$ is bounded, we may find two sequences $\left(\lambda_{1 j}\right)_{j=1}^{\infty},\left(\lambda_{2 j}\right)_{j=1}^{\infty}$ in $]-t_{A}, t_{A}[$ and two vectors $\phi, \psi$ in $X$, such that

$$
\lim _{j} \lambda_{1 j}=t_{A}, \quad \lim _{j} \lambda_{2 j}=-t_{A},
$$


and the vector sequences $\left(x\left(\lambda_{1 j}\right)\right)_{j=1}^{\infty},\left(x\left(\lambda_{2 j}\right)\right)_{j=1}^{\infty}$ converge weakly to $\phi$ and $\psi$, respectively. Taking weak limits in the equality $(I-\lambda A) x(\lambda)=\lambda \varphi$, we get

$$
\left(I-t_{A} A\right) \phi=t_{A} \varphi, \quad\left(I+t_{A} A\right) \psi=-t_{A} \varphi .
$$

Therefore, $\varphi \in \operatorname{Range}\left(I-t_{A} A\right) \cap \operatorname{Range}\left(I+t_{A} A\right)$.

Theorem 1. $\mathcal{R}_{4}(X)$ is dense in $\mathcal{P}_{4}(X)$.

Proof. Let $P$ be an arbitrary element of $\mathcal{P}_{4}(X)$ with the standard form given at the beginning. Take $\varepsilon>0$. We assume $P \notin \mathcal{R}_{4}(X)$ and so, after Lemma 3 , the operators $I-t_{A} A, I+t_{A} A$ are both one-to-one. We know that at least one of these operators must not be onto, otherwise either $I-r_{A} A$, or $I-s_{A} A$, would be a topological isomorphism thus contradicting the definitions of $r_{A}$ and $s_{A}$. Hence, Range $\left(I-t_{A} A\right) \cap \operatorname{Range}\left(I+t_{A} A\right) \neq X$ and so it has empty interior. Thus, we can get hold of a vector $\varphi_{\varepsilon} \notin \operatorname{Range}(I-$ $\left.t_{A} A\right) \cap \operatorname{Range}\left(I+t_{A} A\right)$ such that $\left\|\varphi-\varphi_{\varepsilon}\right\| \leq \varepsilon / 2$. Finally, if we consider the polynomials

$$
\begin{aligned}
Q_{\varepsilon}(x) & :=\langle A x, x\rangle+2\left\langle\varphi_{\varepsilon}, x\right\rangle+k, \\
P_{\varepsilon}(x) & :=\left(1-\|x\|^{2}\right) Q_{\varepsilon}(x),
\end{aligned}
$$

it is clear, after Lemma 4, that $P_{\varepsilon} \in \mathcal{R}_{4}(X)$ and, since, for $\|x\| \leq 1$,

$$
\begin{aligned}
\left|P(x)-P_{\varepsilon}(x)\right| & =\left(1-\|x\|^{2}\right) \cdot\left|Q(x)-Q_{\varepsilon}(x)\right| \\
& =2\left(1-\|x\|^{2}\right) \cdot\left|\left\langle\varphi-\varphi_{\varepsilon}, x\right\rangle\right| \\
& \leq 2\left\|\varphi-\varphi_{\varepsilon}\right\| \leq \varepsilon,
\end{aligned}
$$

it follows that $\left\|P-P_{\varepsilon}\right\| \leq \varepsilon$.

Corollary 1 (Approximate Rolle's Theorem for polynomials of degree four). If $P$ is a continuous polynomial of degree four that vanishes in the unit sphere of the real Hilbert space $X$, then, for each $\varepsilon>0$, there exists $x_{\varepsilon} \in X$ such that $\left\|x_{\varepsilon}\right\|<1$ and $\left\|P^{\prime}\left(x_{\varepsilon}\right)\right\| \leq \varepsilon$.

Proof. Assuming $P \in \mathcal{P}_{4}(X) \backslash \mathcal{R}_{4}(X)$ has the standard form, and proceeding as in the previous theorem, we find a vector $\varphi_{\varepsilon}$ which does not belong to the subspace $\operatorname{Range}\left(I-t_{A} A\right) \cap \operatorname{Range}\left(I+t_{A} A\right)$, and $\left\|\varphi-\varphi_{\varepsilon}\right\| \leq \varepsilon / 6$. 
The polynomial $P_{\varepsilon}$ there defined is in $\mathcal{R}_{4}(X)$, so there exists $x_{\varepsilon} \in X$ with $\left\|x_{\varepsilon}\right\|<1$ and $P_{\varepsilon}^{\prime}\left(x_{\varepsilon}\right)=0$. Hence

$$
\begin{aligned}
\left\|P^{\prime}\left(x_{\varepsilon}\right)\right\|= & \left\|P^{\prime}\left(x_{\varepsilon}\right)-P_{\varepsilon}^{\prime}\left(x_{\varepsilon}\right)\right\| \\
= & \|-2 Q\left(x_{\varepsilon}\right) x_{\varepsilon}+\left(1-\left\|x_{\varepsilon}\right\|^{2}\right)\left(2 A x_{\varepsilon}+2 \varphi\right)+2 Q_{\varepsilon}\left(x_{\varepsilon}\right) x_{\varepsilon} \\
& \quad-\left(1-\left\|x_{\varepsilon}\right\|^{2}\right)\left(2 A x_{\varepsilon}+2 \varphi_{\varepsilon}\right) \| \\
\leq & 2\left\|Q-Q_{\varepsilon}\right\|+2\left\|\varphi-\varphi_{\varepsilon}\right\| \leq 6\left\|\varphi-\varphi_{\varepsilon}\right\| \leq \varepsilon .
\end{aligned}
$$

\section{§3. A Characterization of the Shkarin Polynomials which do not Satisfy Rolle's Theorem}

In [4], we introduced the class of Shkarin polynomials, giving a sufficient condition for those polynomials not to satisfy Rolle's Theorem. We show here that this condition is also necessary. We reproduce the definition of a Shkarin polynomial.

Definition 1. If $P \in \mathcal{P}_{4}(X)$ has the standard form given in the first section, it is then said to be a Shkarin polynomial whenever it satisfies the following conditions:

1. $A$ is a strictly positive operator in $X$, i.e., $x \neq 0$ implies $\langle A x, x\rangle>0$.

2. With the terminology introduced in Section 2, we know that $r_{A}=\frac{1}{\|A\|}$, $s_{A}=-\infty$. We assume in this condition that there is $0<\rho<1$ such that $\|x(\lambda)\| \leq \rho, \quad \lambda<\frac{1}{\|A\|}$.

3. $\|A\|$ is not an eigenvalue, i.e., the operator $I-\frac{1}{\|A\|} A$ is one-to-one.

4. $\varphi$ is not in the range of $I-\lambda A, \lambda>\frac{1}{\|A\|}$.

Lemma 5. If $P$ is a Shkarin polynomial and $P \notin \mathcal{R}_{4}(X)$, then there are vectors $\psi, \phi$ such that, in $X$,

$$
\lim _{\lambda \rightarrow-\infty} x(\lambda)=\psi, \quad \lim _{\lambda \rightarrow 1 /\|A\|} x(\lambda)=\phi .
$$

Proof. Since the set $\{x(\lambda): \lambda \in]-\infty, \frac{1}{\|A\|}[\}$ is bounded, proceeding as in Lemma 4, we find two scalar sequences $\left(\lambda_{1 j}\right)_{j=1}^{\infty},\left(\lambda_{2 j}\right)_{j=1}^{\infty}$ and two vectors $\psi, \phi$, such that

$$
\lim _{j} \lambda_{1 j}=-\infty, \quad \lim _{j} \lambda_{2 j}=\frac{1}{\|A\|},
$$


and the vector sequences $\left(x\left(\lambda_{1 j}\right)\right)_{j=1}^{\infty},\left(x\left(\lambda_{2 j}\right)\right)_{j=1}^{\infty}$ converge weakly to $\psi, \phi$, respectively. Now, since $\|x(\lambda)\|^{2}$ is decreasing in $]-\infty, 0[$, it follows that $\lim _{\lambda \rightarrow-\infty}\|x(\lambda)\|^{2}$ exists.

Moreover, making use of L'Hôpital's rule, the fact that $A$ is self-adjoint and that $A \psi=-\varphi,\left(I-\frac{1}{\|A\|} A\right) \phi=\frac{1}{\|A\|} \varphi$, we have

$$
\begin{aligned}
\lim _{\lambda \rightarrow-\infty}\langle\psi, x(\lambda)\rangle=\lim _{\lambda \rightarrow-\infty}\langle\psi, \lambda(A x(\lambda)+\varphi)\rangle \\
\quad=\lim _{\lambda \rightarrow-\infty} \frac{\langle\varphi, \psi-x(\lambda)\rangle}{1 / \lambda}=\lim _{\lambda \rightarrow-\infty} \frac{\left\langle\varphi, x^{\prime}(\lambda)\right\rangle}{1 / \lambda^{2}} \\
\quad=\lim _{\lambda \rightarrow-\infty} \lambda^{2}\left\langle\varphi, x^{\prime}(\lambda)\right\rangle=\lim _{\lambda \rightarrow-\infty}\|x(\lambda)\|^{2} .
\end{aligned}
$$

But, since $\left(x\left(\lambda_{1 j}\right)\right)_{j=1}^{\infty}$ converges weakly to $\psi$,

$$
\|\psi\|^{2}=\lim _{j}\left\langle\psi, x\left(\lambda_{1 j}\right)\right\rangle=\lim _{\lambda \rightarrow-\infty}\|x(\lambda)\|^{2} .
$$

Hence, convergence in norm is now clear

$$
\lim _{\lambda \rightarrow-\infty}\|\psi-x(\lambda)\|^{2}=\lim _{\lambda \rightarrow-\infty}\left(\|\psi\|^{2}-2\langle\psi, x(\lambda)\rangle+\|x(\lambda)\|^{2}\right)=0 .
$$

Similarly, if $\lambda \in] 0, \frac{1}{\|A\|}[$, since

$$
x(\lambda)=\sum_{n=0}^{\infty} \lambda^{n+1} A^{n} \varphi, \quad x^{\prime}(\lambda)=\sum_{n=0}^{\infty}(n+1) \lambda^{n} A^{n} \varphi,
$$

it follows that

$$
\frac{d}{d \lambda}\|x(\lambda)\|^{2}=2 \sum_{m, n=0}^{\infty}(n+1) \lambda^{m+n+1}\left\langle A^{m+n} \varphi, \varphi\right\rangle>0,
$$

and so the function $\|x(\lambda)\|^{2}$ increases in $] 0, \frac{1}{\|A\|}\left[\right.$ and thus $\lim _{\lambda \rightarrow \frac{1}{\|A\|}}\|x(\lambda)\|^{2}$ exists. Now

$$
\begin{aligned}
& \langle\varphi, \phi-x(\lambda)\rangle=\|A\|\left\langle\left(I-\frac{A}{\|A\|}\right) \phi, \phi-x(\lambda)\right\rangle \\
& =\|A\|\left(\|\phi\|^{2}-\langle\phi, x(\lambda)\rangle-\frac{1}{\|A\|}\langle A \phi, \phi\rangle+\frac{1}{\|A\|}\langle A \phi, x(\lambda)\rangle\right) \\
& =\|A\|\left(\frac{1}{\|A\|}\langle\phi, A x(\lambda)+\varphi\rangle-\langle\phi, x(\lambda)\rangle\right) \\
& =\|A\|\left(\frac{1}{\lambda\|A\|}-1\right)\langle\phi, x(\lambda)\rangle=\frac{1-\lambda\|A\|}{\lambda}\langle\phi, x(\lambda)\rangle .
\end{aligned}
$$


Therefore

$$
\begin{aligned}
& \lim _{\lambda \rightarrow \frac{1}{\|A\|}}\langle\phi, x(\lambda)\rangle=\lim _{\lambda \rightarrow \frac{1}{\|A\|}} \frac{\langle\varphi, \phi-x(\lambda)\rangle}{1 / \lambda-\|A\|} \\
& \quad=\lim _{\lambda \rightarrow \frac{1}{\|A\|}} \frac{\left\langle\varphi, x^{\prime}(\lambda)\right\rangle}{1 / \lambda^{2}}=\lim _{\lambda \rightarrow \frac{1}{\|A\|}}\|x(\lambda)\|^{2} .
\end{aligned}
$$

Finally

$$
\|\phi\|^{2}=\lim _{j}\left\langle\phi, x\left(\lambda_{2 j}\right)\right\rangle=\lim _{\lambda \rightarrow \frac{1}{\|A\|}}\|x(\lambda)\|^{2},
$$

which implies that

$$
\lim _{\lambda \rightarrow \frac{1}{\|A\|}}\|\phi-x(\lambda)\|^{2}=0
$$

The following characterization clearly improves the result given in [4, Theorem 1], which we reproduce here

Theorem. If $P(x)$ is a Shkarin polynomial such that

$$
\|\phi\|^{2}=\lim _{\lambda \rightarrow \frac{1}{\|A\|}}\|x(\lambda)\|^{2},
$$

then $P(x)$ does not satisfy Rolle's Theorem if and only if

$$
\langle A \psi, \psi\rangle<k<\|A\|\left(1-3\|\phi\|^{2}\right)+\langle A \phi, \phi\rangle .
$$

Theorem 2. Let $P$ be a Shkarin polynomial. Then, $P$ does not satisfy Rolle's Theorem if and only if the following inequality holds

$$
\langle A \psi, \psi\rangle<k<\langle A \phi, \phi\rangle+\|A\|\left(1-3\|\phi\|^{2}\right) .
$$

Proof. For the sufficiency part we refer to Proposition 1 of [4]. Seeking necessity, notice that $\langle A \psi, \psi\rangle \neq k$, otherwise $0=Q(\psi)=P^{\prime}(\psi)$ and, by condition 2 in the definition of a Shkarin polynomial, it follows that $\|\psi\| \leq$ $\rho<1$. Assuming $k<\langle A \psi, \psi\rangle$, then, on one hand we know that $h(\lambda)<1$, $\lambda \in]-\infty, \frac{1}{\|A\|}[$, but, on the other hand, after Lemma 5 ,

$$
\begin{aligned}
\lim _{\lambda \rightarrow-\infty} h(\lambda) & =2\|\psi\|^{2}+\lim _{\lambda \rightarrow-\infty} \lambda(\langle\varphi, x(\lambda)\rangle+k) \\
& =2\|\psi\|^{2}+(-\infty)(k-\langle A \psi, \psi\rangle) \\
& =+\infty,
\end{aligned}
$$


which contradicts the boundedness of $h(\lambda)$. Thus $k>\langle A \psi, \psi\rangle$. For the other part of the inequality, again using Lemma 5 , we know that, by setting $x\left(\frac{1}{\|A\|}\right):=\phi$, we obtain a continuous extension of $h(\lambda)$ to the closed interval ] $\left.-\infty, \frac{1}{\|A\|}\right]$. Since $P \notin \mathcal{R}_{4}(X)$, we must have that $h\left(\frac{1}{\|A\|}\right)<1$. Hence

$$
\begin{aligned}
1 & >h\left(\frac{1}{\|A\|}\right)=\lim _{\lambda \rightarrow \frac{1}{\|A\|}} h(\lambda)=\lim _{\lambda \rightarrow \frac{1}{\|A\|}}\left[2\|x(\lambda)\|^{2}+\lambda(\langle\varphi, x(\lambda)\rangle+k)\right] \\
& =2\|\phi\|^{2}+\frac{\langle\varphi, \phi\rangle+k}{\|A\|}=3\|\phi\|^{2}+\frac{k-\langle A \phi, \phi\rangle}{\|A\|},
\end{aligned}
$$

and the inequality follows.

\section{References}

[1] Aron, R. M., Hervés, C. and Valdivia, M., Weakly continuous mappings on Banach spaces, J. Funct. Anal., 52 (1983), 189-204.

[2] Azagra, D., Gómez, J. and Jaramillo, J. A., Rolle's Theorem and the negligibility of points in infinite-dimensional Banach spaces, J. Math. Anal. Appl., 213 (1997), 487495.

[3] Ferrer, J., On Rolle's Theorem in spaces of infinite dimension, Indian J. Math., 42 (2000), 21-36.

[4] , Rolle's Theorem for polynomials of degree four in a Hilbert space, J. Math. Anal. Appl., 265 (2002), 322-331.

[5] Shkarin, S. A., On Rolle's Theorem in infinite-dimensional Banach spaces, Mat. Zametki, 51 (1992), 128-136. 